\title{
Eletroconvulsoterapia na terapêutica da mania em paciente com hidrocefalia
}

\author{
Eletroconvulsotherapy in the treatment of patient \\ with mania and hydrocephalus \\ Izabela G. Barbosa', Fábio L. Rocha'
}

\section{RESUMO}

A evidência de segurança e eficácia do emprego de eletroconvulsoterapia (ECT) no tratamento de transtornos psiquiátricos em pacientes com distúrbios neurológicos é fundamentada, em geral, em relatos de casos. Em relação ao emprego em pacientes com hidrocefalia, há apenas oito casos descritos: seis abordando o tratamento de episódios depressivos, um para estado catatônico e um relato em paciente com auto-agressão. Os autores descrevem o caso de um paciente com 20 anos de idade, diagnóstico de hidrocefalia aos 12 anos, internado em ala psiquiátrica com episódio maníaco com sintomas psicóticos. Houve remissão completa da sintomatologia após quatro sessões de ECT. Não houve deterioração neurológica ou outros efeitos colaterais. O resultado sugere que a ECT é segura e eficaz no tratamento de episódios maníacos em pacientes com hidrocefalia.

\section{Palavras-chave \\ Eletroconvulsoterapia, hidrocefalia, transtorno bipolar, mania.}

\section{Keywords}

Electroconvulsive therapy, hydrocephalus, bipolar disorder, mania.

\begin{abstract}
Safety and efficacy evidence on the use of electroconvulsive therapy (ECT) in the treatment of psychiatric patients with neurological diseases is generally based on case reports. In relation to the use of ECT on patients with hydrocephalus, there are only eight case reports: six for depressive episodes, one for catatonic state and one for self-aggression. In this article the authors describe the case of a 20-year-old male patient, with a hydrocephalus diagnosis since the age of 12, admitted to a psychiatric hospital suffering from a manic episode with psychotic symptoms. There was complete remission of symptoms after four sessions of ECT, with no neurological damage or other side effects. The result suggests that ECT is safe and effective in treating manic episodes in patients with hydrocephalus.
\end{abstract}

A eletroconvulsoterapia (ECT) é tratamento comprovadamente seguro e eficaz, utilizado em vários transtornos psiquiátricos. É considerada a abordagem mais eficaz nos estados depressivos ${ }^{1}$. A ECT é também empregada no tratamento de episódios maníacos, em exacerbações psicóticas da esquizo- frenia, nos transtornos esquizoafetivo e esquizofreniforme, em quadros catatônicos, no transtorno obsessivo-compulsivo e na síndrome neuroléptica maligna². Considera-se a ECT segura em pacientes idosos, em portadores de problemas cardiovasculares e em mulheres grávidas ${ }^{2-4}$.

1 Instituto de Previdência dos Servidores do Estado de Minas Gerais (IPSEMG).

Recebido em

21/7/2008

Aprovado em

20/10/2008

Endereço para correspondência: Izabela G. Barbosa

Av. dos Andradas, 2287, sala 1105 - 30120-010 - Belo Horizonte, MG

E-mail: izabela_g_b@yahoo.com 
Os principais efeitos colaterais da ECT são alterações cognitivas (amnésia anterógrada, retrógrada e estado confusional agudo), cefaléia, dores musculares, náuseas, anorexia, convulsões prolongadas e status epilépticos ${ }^{2-4}$.

Não existem contra-indicações absolutas ao emprego da ECT. A taxa de mortalidade é similar aos procedimentos que envolvam anestesia geral, ou seja, cerca de um em cada 80 mil casos tratados. Os eventos que resultaram morte estão relacionados a complicações cardiovasculares e pulmonares ${ }^{2-4}$.

A ECT também pode ser utilizada em pacientes com doenças neurológicas, seja para o tratamento de transtornos psiquiátricos, seja para o alívio de sintomas da própria doença neurológica. Por exemplo, há relatos de eficácia em depressão associada a quadros demenciais, em delirium de etiologia diversa, na doença de Parkinson (sintomas de humor e motores), assim como em transtornos de movimento ${ }^{5}$. Nesses casos, a evidência de segurança e eficácia é fundamentada, em geral, em relatos e séries de casos. Na maioria das vezes, os efeitos colaterais não são relatados de maneira consistente ${ }^{5}$.

Entre os relatos de casos envolvendo o emprego de ECT em pacientes com problemas neurológicos, há número muito reduzido sobre o uso em pacientes com hidrocefalia, envolvendo quadros de depressão, catatonia e auto-agressão ${ }^{6-13}$. Os autores descrevem o caso de um paciente com hidrocefalia, tratado de episódio maníaco com ECT.

\section{RELATO DE CASO}

Paciente com 20 anos, sexo masculino, o grau, solteiro, hospitalizado com quadro psiquiátrico iniciado há três dias, caracterizado por agitação psicomotora intensa, agressividade, idéias delirantes de grandeza e perseguição, insônia e logorréia.

Em sua história médica constava diagnóstico de hidrocefalia realizado aos 12 anos. A hidrocefalia foi descoberta acidentalmente, em atendimento de pronto-socorro, após atropelamento. Nos oito anos até a internação psiquiátrica, o paciente foi avaliado periodicamente por neurologista. Não houve descompensação da hidrocefalia e o paciente manteve-se assintomático. Não houve necessidade de cirurgia para derivação. De acordo com os familiares, a hidrocefalia era congênita. Não havia história de abuso ou dependência de álcool ou de uso de drogas. Sem outros antecedentes psiquiátricos. Na história familiar, constava depressão em prima e quadro psiquiátrico sem maiores informações em tia-avó.

Os exames clínico e neurológico não mostraram alterações. Especificamente, não havia incontinência urinária ou alterações na marcha ou sintomas de hipertensão intracraniana, como cefaléia, vômitos, tinido, letargia e alterações visuais. O exame de fundo de olho também não evidenciou alterações patológicas. Os exames laboratoriais à admissão não mostraram alterações significativas. A tomografia cerebral evidenciou apenas hidrocefalia, sem calcificações ou outras alterações. Foram excluídas doenças que pudessem cursar com quadro psiquiátrico semelhante (hipertireoidismo e doença de Wilson) e descompensação da hidrocefalia. A hipótese diagnóstica foi de transtorno bipolar, episódio maníaco com sintomas psicóticos (DSM IV).

Em virtude da agitação psicomotora intensa, foi necessário o uso de fármacos injetáveis por via intramuscular, conforme descrição:

- dia 1: haloperidol (20 mg), prometazina (150 mg) e midazolam (15 mg);

- dia 2: haloperidol (20 mg), prometazina (200 mg) e acetato de zuclopentixol (50 mg); midazolam (30 mg);

- dia 3: haloperidol (40 mg) e prometazina (250 mg);

- dia 4: haloperidol (10 mg), prometazina (100 mg) e acetato de zuclopentixol (100 mg);

- dia 5: haloperidol (10 mg), prometazina (100 mg), acetato de zuclopentixol (100 mg) e levomepromazina (25 mg).

Com o emprego de psicofármacos injetáveis, a redução da agitação psicomotora era apenas transitória. No quinto dia, o paciente iniciou elevação da temperatura corporal e parkinsonismo. Os exames laboratoriais evidenciaram aumento da creatina fosfoquinase (CPK) e de enzimas hepáticas. Em virtude da gravidade do quadro clínico, da resposta insuficiente aos medicamentos e da ocorrência de efeitos colaterais, propôs-se a realização de ECT. O aparelho utilizado foi o Thymatron DG (Somatics LLC, Lake Bluff, IL). No procedimento anestésico foram utilizados cloreto de succinilcolina e tiopental. Os eletrodos foram posicionados bilateralmente. A carga foi determinada pela idade do paciente. As aplicações foram monitorizadas por eletroencefalografia e por acompanhamento das contrações em membro garroteado. As sessões de ECT e a utilização de fármacos injetáveis para controle de agitação são relatados a seguir:

- dia 6: primeira sessão de ECT com carga de 20\% e duração de convulsão motora de 16 segundos. Após 1 minuto de hiperventilação, o paciente foi submetido a novo estímulo, com carga de $40 \%$ e com convulsão clínica de 28 segundos. No mesmo dia, acetato de zuclopentixol (50 mg);

- dia 7: midazolam (15 mg);

- dia 8: segunda sessão de ECT, com carga de 40\% e duração de convulsão motora de 28 segundos. No mesmo dia, midazolam (30 mg);

- dia 11: terceira sessão de ECT, carga de 40\% e duração de convulsão motora de 29 segundos;

- dia 13: quarta e última sessão de ECT, carga de 35\% e duração de convulsão motora de 27 segundos. 
A partir do nono dia, não houve mais necessidade de medicamentos para agitação psicomotora. No $13^{\circ}$ dia, última aplicação de ECT, o paciente apresentava-se eutímico e sem sintomas psicóticos. Não houve deterioração neurológica ou outros efeitos colaterais em virtude da ECT. No $14^{\circ}$ dia, foi iniciado o uso de ácido valpróico, medicação com a qual o paciente recebeu alta hospitalar.

\section{DISCUSSÃO}

De acordo com o conhecimento dos autores, este é o primeiro relato de emprego da ECT para tratamento de episódio maníaco com sintomas psicóticos em paciente portador de hidrocefalia. Houve remissão completa da sintomatologia, sem deterioração neurológica ou outros efeitos colaterais.

Há oito relatos prévios do emprego da ECT em pacientes com hidrocefalia: seis para o tratamento de depressão ${ }^{6-10,13}$, um para catatonia' ${ }^{12}$ e um para quadro de auto-agressão de origem orgânica"1. Houve melhora em todos, exceto no quadro de auto-agressão.

Em relação à tolerabilidade nos oito casos descritos, um não aborda o tema, em dois não houve efeitos adversos e em cinco foram descritos efeitos colaterais, mais frequentemente desorientação (Tabela 1). Esses efeitos foram transitórios. Com base nesses relatos, pode-se considerar a ECT em pacientes com hidrocefalia terapêutica razoavelmente segura. Entretanto, para minimizar a ocorrência de alterações cognitivas, recomenda-se o emprego de número reduzido de sessões (três a quatro) ${ }^{6}$.

Os casos relatados referem-se a pacientes com hidrocefalia de pressão normal, isto é, não se tem experiência com hidrocefalia descompensada. Entretanto, há consenso na literatura de que a presença de hipertensão craniana de qualquer origem é contra-indicação relativa para o emprego da $\mathrm{ECT}^{2}$. Consequentemente, pacientes com história de hidrocefalia devem ser avaliados por meio de fundoscopia para detecção de sinais de hipertensão intracraniana. Um potencial aumento de risco ocorreria, também, com o emprego de succinilcolina na indução anestésica dos pacien- tes com hidrocefalia. Experimentos em animais evidenciaram aumento da pressão intracraniana com o uso dessa substância ${ }^{14}$. Entretanto, não se detectaram problemas com o emprego da succinilcolina em humanos com doenças neurológicas ${ }^{15}$. Também, no presente relato, o emprego da succinilcolina não acarretou complicações.

Outros aspectos a serem considerados no caso apresentado são o rápido início de ação da ECT e a lise da sintomatologia com pequeno número de sessões em paciente com episódio maníaco de difícil manejo com psicofármacos injetáveis, seja por respostas muito fugazes, seja pelo desenvolvimento de efeitos colaterais.

Um outro aspecto que pode ser discutido no presente relato é o diagnóstico psiquiátrico. $O$ paciente apresentouse com síndrome maniforme à internação. Duas hipóteses podem ser consideradas: transtorno orgânico do humor ou transtorno bipolar, episódio maníaco. Os autores optaram pelo diagnóstico de transtorno bipolar por tratar-se de quadro de hidrocefalia compensada em oito anos de acompanhamento neurológico, sem sinais de descompensação durante o episódio maniforme - os exames clínico e neurológico não evidenciaram alterações. Particularmente, não havia cefaléia, vômitos, tinido, letargia, alterações visuais e papiledema, sintomas comuns em hipertensão intracraniana' ${ }^{16}$.

\section{CONCLUSÃO}

A ECT parece ser eficaz no tratamento de episódios de humor, depressão ou mania, em pacientes com hidrocefalia. Há relatos prévios de eficácia em depressão e catatonia. No presente relato, houve remissão completa de episódio maníaco. Também não houve efeitos colaterais. A ECT parece ser razoavelmente segura para emprego em pacientes com hidrocefalia compensada ou hidrocefalia de pressão normal. Os efeitos colaterais descritos na literatura, como desorientação e outras alterações cognitivas, são transitórios. Entretanto, cuidado especial deve ser tomado com pacientes com hidrocéfalo descompensado, já que hipertensão intracraniana de qualquer origem é considerada contra-indicação relativa à ECT.

Tabela 1. Relatos de caso do emprego de ECT em pacientes com hidrocefalia.

\begin{tabular}{|c|c|c|c|}
\hline Autores & Diagnóstico & Comorbidade neurológica & Complicações \\
\hline Tsuang et al. (1979) ${ }^{6}$ & Depressão & Hidrocefalia de pressão normal com derivação ventriculojugular & Desorientação e incontinência fecal e urinária \\
\hline Mansheim $(1983)^{7}$ & Depressão & Hidrocefalia com derivação ventriculogástrica & Ausentes \\
\hline Levy e Levy $(1987)^{8}$ & Depressão & Hidrocefalia de pressão normal com derivação ventriculoperitoneal & Desorientação profunda \\
\hline Coffey et al. $(1987)^{9}$ & Depressão & Hidrocefalia de pressão normal com derivação ventriculoatrial & Desorientação prolongada \\
\hline Cardno e Simpson $(1991)^{10}$ & Depressão & Hidrocefalia com derivação & Ausentes \\
\hline Mashiko et al. (1996)"11 & Agressividade & Hidrocefalia sem derivação & Não há descrição de complicações \\
\hline Malur et al. $(2001)^{12}$ & Catatonia & Hidrocefalia comunicante & Déficit cognitivo \\
\hline Hanreta e Malek-Ahmadi (2001) ${ }^{13}$ & Depressão & Hidrocefalia de pressão normal com derivação ventriculoperitoneal & Agitação pós-ECT \\
\hline
\end{tabular}




\section{Conflitos de interesse}

Não há conflito de interesses.

\section{REFERÊNCIAS}

1. The UK ECT Review Group. Efficacy and safety of electroconvulsive therapy in depressive disorder: a systematic review and meta-analysis. Lancet. 2003;361:799-808.

2. Teixeira PJR. Atualização em eletroconvulsoterapia. J Bras Psiquiatria. 1997;46(11):601-9.

3. Rocha FL, Cunha UGV. A eletroconvulsoterapia no tratamento da depressão do idoso. Rev. ABP-APAL. 1992;14(1):2-8.

4. Rocha FL. A eletroconvulsoterapia (ECT) no tratamento da depressão em pacientes portadores de problemas cardiovasculares: atualização. J Bras Psiquiatr. 1993;42(2):89-95.

5. Trimble MR, Krishnamoorthy ES. The use of ECT in neuropsychiatric disorders. In: Scott AIF (editor). The ECT Handbook. 2. ed. London. 2004; p. 47-56.

6. Tsuang MT, Tindball JS, Geller D. ECT in a depressed patient with shunt in place for normal pressure hydrocephalus. Am J Psychiatry. 1979;136 1205-6.

7. Mansheim P. ECT in the treatment of a depressed adolescent with meningomyelocele, hydrocephalus and seizure. J Clin Psychiatry. 1983;3:302-6.
8. Levy SD, Levy SB. Electroconvulsive therapy in two neurosurgical patients: skull prothesis and ventricular shunt. Convuls Ther. 1987;3:46-8.

9. Coffey CE, Hoffman G, Weiner RD, Moossy JJ. Electroconvulsive therapy in a depressed patient with a functioning ventriculoatrial shunt. Convuls Ther. 1987;3:302-6.

10. Cardno AG, Simpson CJ. Electroconvulsive therapy in Paget's disease and hydrocephalus. Convuls Ther. 1991;7:48-51.

11. Mashiko H, Yokoyama H, Matsumoto H, Niwa S. Trazodone for agression in an adolescent with hydrocephalus. Psychiatry Clin Neurosci. 1996;50:133-136.

12. Malur C, Pasol E, Francis A. ECT for prolonged catatonia. J ECT. 2001;17:55-9.

13. Hanreta AL, Malek-Ahmadi. Successful ECT in a patient with hydrocephalus, shunt, hypopituitarism, and paraplegia. J ECT. 2001;17:71-4.

14. Lanier WL, Middle JH, Michenfelder JD. Cerebral stimulation following succinylcholine in dogs. Anesthesiology. 1986;64:551-9.

15. Kovarik WD, Mayberg TS, Lam LA, Mathisen TL, Winn HR. Succinylcholine does not change intracranial pressure, cerebral blood flow, or the encephalogram in patients with neurologic injury. Anesth Analg. 1994;78:469-73.

16. DeAngelis LM. Tumores do sistema nervoso central e hipertensão e hipotensão intracranianas. In: Goldman L, Ausiello D, editores. Cecil Tratado de Medicina Interna. Rio de Janeiro: Elsevier Press; 2005. p. 2744-56. 